\title{
Infra-red Thermal Imaging of the Inner Canthus: Correlates with the Temperature of the Injured Human Brain
}

\author{
Charmaine Childs ${ }^{1}$, Mya Myint Zu ${ }^{1}$, Aung Phyo Wai ${ }^{1}$, Yeo Tseng Tsai ${ }^{1}$, Shiqian Wu ${ }^{2}$, Wang Li ${ }^{3}$ \\ ${ }^{1}$ ALCNS and Department of Surgery, National University of Singapore (NUS), National University Hospital (NUH), Singapore \\ ${ }^{2}$ Signal Processing Department, Institute for Infocomm Research, Fusionopolis Way, \#21-01 Connexis, Singapore \\ ${ }^{3}$ National Metrology Centre (NMC), A*Star, Singapore \\ Email: nurcc@nus.edu.sg
}

Received 2012

\begin{abstract}
Introduction: Infra-red (IR) thermometry is a safe and valid method to determine internal and surface temperature in human subjects. Under conditions of brain damage (head injury or stroke) knowledge of changes in the temperature of intracranial tissue is justified because of the vulnerability of neurons to accelerated damage at temperatures at the upper end of the febrile range. Aim: To determine the temperature at the inner canthus (IC) of the eye as a potential surrogate for brain temperature. Methods: Invasive monitoring of deep brain structures, lateral ventricle and deep white matter. IR temperature readings obtained at right and left IC. Results: Strong correlations were evident between R and L IC and brain. Close, as well as poor, agreement between sites was shown in some patients and at some times. For right hemispheric lesions four had a better correlation between TbrV and TRIC when compared to TLIC. When the correlation between TbrV and TLIC was better compared to TbrV and TRIC, four had a predominant right hemispheric lesion. Conclusions: Improved techniques for IR thermal imaging accuracy at the bedside has the potential to improve temperature measurement agreement. The predominant lesion side may have a bearing on maximum ipsilateral IC temperature Further studies are ongoing in this pilot study population.
\end{abstract}

Keywords: Brain Temperature; Infra-red Thermometry; Inner Canthus; Thermal Imaging; Eye

\section{Introduction}

Temperature changes play a major role in the survival of all animal species [1]. In mammals, body temperature is maintained within a relatively 'tight' range with normal values of the order of $36.7^{\circ} \mathrm{C}$. Nevertheless, differences in the temperature of the viscera are reported, typically the variation is small only $\left(<0.5^{\circ} \mathrm{C}\right)$ [2]. Whilst a rise in deep body (core) temperature due to fever is common after trauma and critical illness it is usually regarded as an adaptive response with potential survival benefit [3]. By contrast, if damage to brain occurs due to ischaemia (stroke) or trauma (mild to moderate traumatic brain injury, TBI) the prevailing view is that even small increases in temperature will accelerate secondary damage of vulnerable ischaemic neurons; the consequence being an increased risk of death and worsened neurological outcome [4].

Knowing when a rise in brain temperature has occurred is clinically important. It is generally assumed that body temperature is a reliable 'surrogate' for brain temperature but proxy measures for brain temperature are often unreliable [5]. Direct monitoring is safe and recommended during neurocritical care [6]. However, it is customary to revert to body measurement sites (rectum, tympanum, oral, tympanic) once the patient leaves the intensive care unit (ICU). Studies from our group have shown the potential of infra-red (IR) thermometry as a proxy for brain temperature measurement. In the normal to febrile temperature range, IR temporal artery measurement is closer to brain temperature than is IR tympanic membrane temperature with average differences of $0.3^{\circ} \mathrm{C}$ only [7]. IR surface temperature measurements at sites other then ear or fore- head thus have potential as a surrogate for brain temperature.

The aim of the current study was to determine if IR thermal imaging of the inner canthus of the eye provides a clinically useful approach to brain temperature estimation in patients with severe intracerebral trauma.

\section{Materials and Methods}

The study was approved by the local research ethics committee. Written informed consent was obtained from the patient's spouse or relative before measurements were taken.

Patients: Adults aged $>21$ years with severe traumatic brain injury (TBI) admitted for neurocritical care were eligible for inclusion in the study. All patients were intubated and sedated and mechanically ventilated and were admitted to the ICU after neurosurgery or as direct admissions to ICU for neuromonitoring. The patients were treated in accordance with local Neurocritical care guidelines and studied for a maximum of 5 days from arrival to ICU.

\subsection{Monitoring}

Invasive brain monitoring: Temperature monitoring was obtained at two brain sites; within brain tissue $\left(\mathrm{T}_{\mathrm{br}} \mathrm{t}\right)$ and within the lateral ventricle $\left(\mathrm{T}_{\mathrm{br}} \mathrm{V}\right)$. Thermal images of facial skin temperature were also obtained.

\subsection{Materials}

$\mathrm{T}_{\mathrm{br}} \mathrm{t}$ was measured using a multiparameter (temperature, pres- 
sure, oxygen sensor) tissue probe (Neurovent-PTO; Raumedic, Munchberg, Germany). The sensor tip was positioned approximately 3-4 cm in deep white matter of the frontal lobe. $\mathrm{T}_{\mathrm{br}} \mathrm{V}$ was measured using a second integrated probe (pressure and temperature sensor; Neurovent-Temp-IFD-S-C; Raumedic, Munchberg, Germany) with sensor tip positioned within the lateral ventricle.

Non-contact IR thermal imaging: Thermal images of the face were obtained using a FLIR Systems Thermographic Camera (Model $\left.{ }^{\mathrm{TM}} \mathrm{T} 365\right)$. The FLIR camera was calibrated to fever screening standards of the National Metrology Centre (NMC), A*STAR, Singapore.

\subsection{Methods}

Camera emissivity was set to 0.98 . At each imaging session, the IR camera was positioned and the focus adjusted to ensure clarity and accuracy of the image and temperature values. The target region of interest (ROI) was temperature of right inner canthus $\left(\mathrm{T}_{\mathrm{RIC}}\right)$ and left inner canthus $\left(\mathrm{T}_{\mathrm{LIC}}\right)$. A series of three consecutive IR images were captured at each measurement interval with patients lying supine. All IR images were taken at the same 'tilt angle' from a standing position at the bedside. To obviate the effect of patient to camera distance on the temperature reading, manual adjustment was made to approximate 1 meter. A FLIR Quick Report 1.2 was used to document temperature $\left({ }^{\circ} \mathrm{C}\right)$ readings from the images. The maximum temperature reading of each ROI (Figure 1) was used in data analysis.

\subsection{Data Analysis}

Data analysis consists of three parts: 1) probability of distribution of FLIR temperature measurements. As random error occurs in measurement, the random measurements follow a specific distribution; generally as a normal distribution; 2) correlation analysis, i.e., to determine whether the readings of $\mathrm{T}_{\mathrm{RIC}} / \mathrm{T}_{\mathrm{LIC}}$ from FLIR images are correlated with brain temperature $\left(\mathrm{T}_{\mathrm{br}} \mathrm{t}\right.$, and $\left.\mathrm{T}_{\mathrm{br}} \mathrm{V}\right)$; 3 ) curve fitting between $\mathrm{T}_{\mathrm{RIC}} / \mathrm{T}_{\mathrm{LIC}}$ and $\mathrm{T}_{\mathrm{br}} \mathrm{t} / \mathrm{T}_{\mathrm{br}} \mathrm{V}$ in order to predict the brain temperature by the measurement $\mathrm{T}_{\mathrm{RIC}} / \mathrm{T}_{\mathrm{LIC}}$.
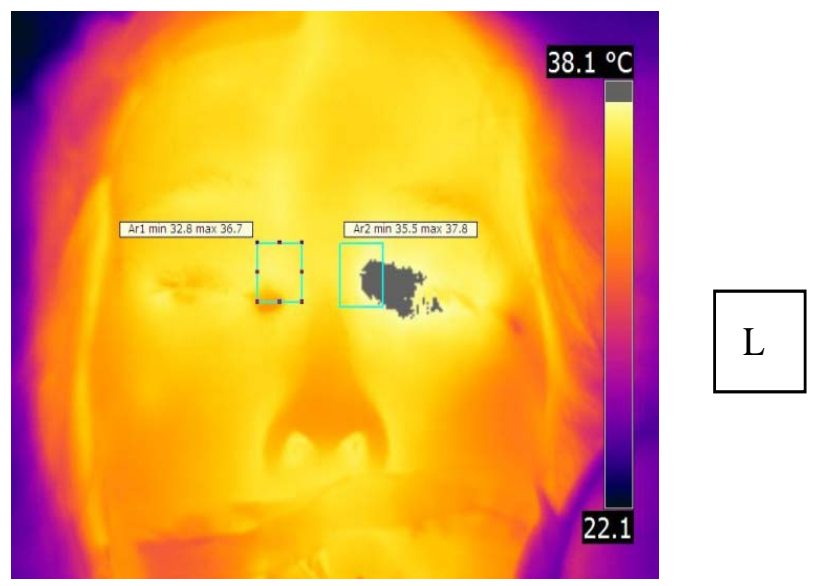

Figure 1. ROI of Inner canthus of patient with severe TBI. L =left side face. Grey colour indicates region of highest temperature values.

\section{Results}

Twelve patients (8 male) aged 21-78 (median 50) years with severe TBI were studied 7-60 (median 16) hours after injury in below thermoneutral ambient conditions, typically of approx $18^{\circ} \mathrm{C}$. Clinical care and nursing procedures in the ICU prevented obtaining a consistent numbers of FLIR images for each patient. FLIR images were taken on 8-55 (median 30) occasions. Every effort was made to obtain 'simultaneous' readings of $\mathrm{T}_{\text {br }} \mathrm{V}$ and $\mathrm{T}_{\text {RIC }} / \mathrm{T}_{\text {LIC }}$ and $\mathrm{T}_{\text {br }} \mathrm{t}$ and $\mathrm{T}_{\mathrm{RIC}} / \mathrm{T}_{\mathrm{LIC}}$. No significant effect of time was observed for FLIR temperature readings with respect to brain temperature. IC temperatures followed a normal distribution (Figure 2).

In 6 of 12 patients the correlation between $\mathrm{T}_{\mathrm{RIC}}$ and $\mathrm{T}_{\mathrm{LIC}}$ was better with respect to ventricular temperature $\left(\mathrm{T}_{\mathrm{br}} \mathrm{V}\right)$ and in 5 patients correlations for $\mathrm{T}_{\mathrm{RIC}}$ and $\mathrm{T}_{\mathrm{LIC}}$ were better for brain tissue temperature $\left(\mathrm{T}_{\mathrm{br}} \mathrm{t}\right)$. In one patient only, the correlations between $\mathrm{T}_{\mathrm{br}} \mathrm{V}$ and $\mathrm{T}_{\mathrm{RIC}}$ and $\mathrm{T}_{\mathrm{br}} \mathrm{V}$ and $\mathrm{T}_{\mathrm{LIC}}$ were comparable.

The predominant lesion side may have a bearing on maximum ipsilateral IC temperature. For right hemispheric lesions $(n=5)$ four had a better correlation between $\mathrm{T}_{\mathrm{br}} \mathrm{V}$ and $\mathrm{T}_{\mathrm{RIC}}$ when compared to $\mathrm{T}_{\text {LIC }}$. When the correlation between $\mathrm{T}_{\text {br }} \mathrm{V}$ and $\mathrm{T}_{\text {LIC }}$ was better compared to $\mathrm{T}_{\text {br }} \mathrm{V}$ with $\mathrm{T}_{\text {RIC }}$ four of five had a predominant right hemispheric lesion. Patients had better outcomes when higher IC values were 'right sided' and worse outcomes when IC values were 'left sided'.

Whilst the correlations between $\mathrm{T}_{b r} \mathrm{~V}$ and RIC and $\mathrm{T}_{\mathrm{br}} \mathrm{V}$ and LIC were strong (for example in patient 5; 0.8208 and 0.7638 respectively) neither IC temperature provided a consistently reliable measure for brain temperature. Figure 3 shows the agreement between brain and R and L IC temperatures with small and large differences between the sites.

For patient 3 , correlations between $\mathrm{T}_{b r} \mathrm{~V}$ and RIC and $\mathrm{T}_{\mathrm{br}} \mathrm{V}$ and LIC were strongly positive $(0.9585$ and 0.9542 respectively). Differences between the sites were $<1.0^{\circ} \mathrm{C}$ for more than half of the measurement intervals (Figure 4).

\section{Discussion}

The medial canthus has a key functional role in providing a

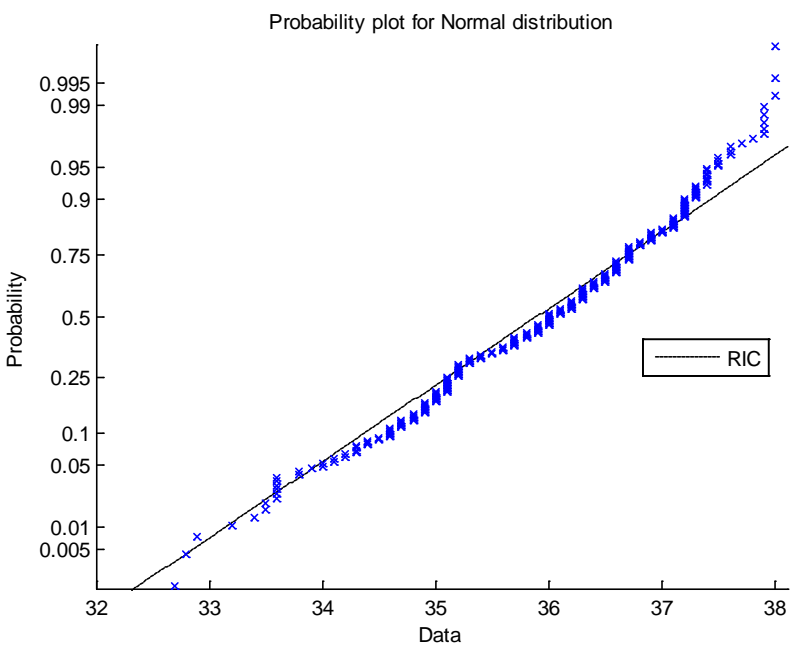

Figure 2. Probability plot shows Normal Distribution of $\mathrm{T}_{\mathrm{RIC}}\left({ }^{\circ} \mathrm{C}\right)$. 


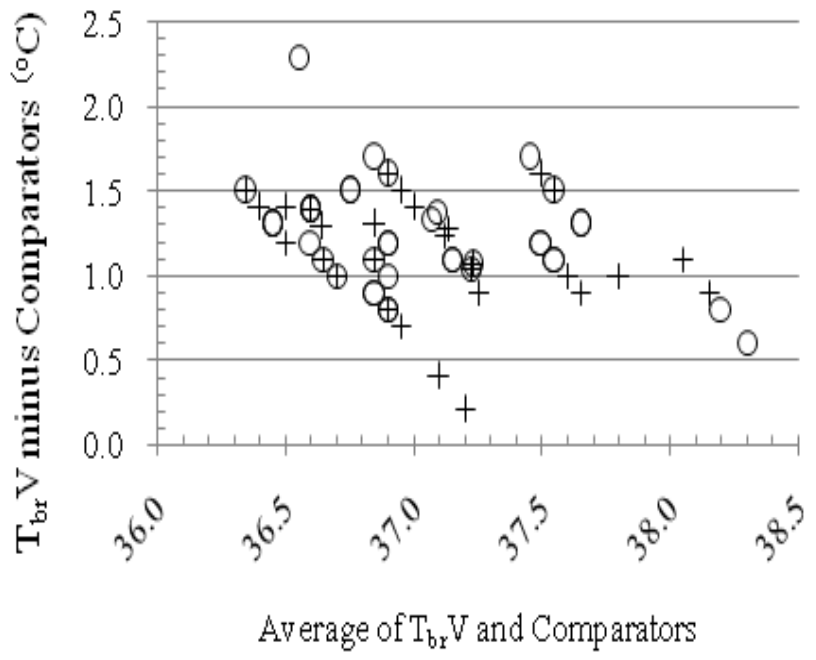

Figure 3. Bland-Altman plot showing temperature differences between ventricular brain temperature $\left(\mathrm{T}_{\mathrm{br}} \mathrm{V}\right)$ and comparators for patient 5. (Comparator: $T_{\text {RIC }}=$ Right Inner Canthus Temperature (+); $\mathbf{T}_{\text {LIC }}=$ Left Inner Canthus Temperature (o).)

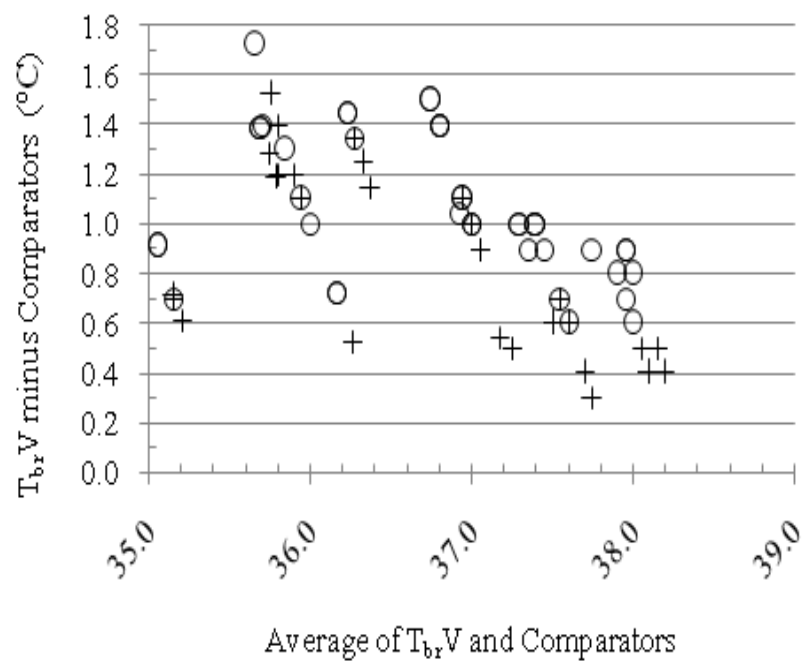

Figure 4. Bland-Altman plot showing temperature differences between ventricular brain temperature $\left(\mathrm{T}_{\mathrm{br}} \mathrm{V}\right)$ and comparators for patient 3. (Comparator: $\mathbf{T}_{\mathrm{RIC}}=$ Right Inner Canthus Temperature (+); $\mathbf{T}_{\text {LIC }}=$ Left Inner Canthus Temperature (o).)

fixed-point fulcrum for the function of the eyelid. Anatomically, the IC has been exploited during mass fever scanning (pandemic infections such as SARS) due to the proximity of the arterial supply at the skin surface. Within this small IC region lie branches of ophthalmic, dorsal nasal, supratrochlear and superior palpebral arteries and angular artery [8]. As the angular artery (giving off three branches) lies directly beneath the skin of the inner canthus and originates from the ophthalmic artery (supratrochlear branch), the dense supply of vessels and thus blood perfusion makes this site a potentially useful spot for estimation of core temperature [9]. In this study we have also obtained pilot data to assess the potential of the IC as a biomarker for anatomical lesion side and TBI outcome.

In previous studies, differences between body (tympanic) and IC temperature deviated significantly, both sites were criticized for their poor reliability in estimation of core temperature. Introducing the oesophageal site to improve the reliability of the 'core' measurement did not reduce the variability of the measurements between sites. In the current study, brain temperature obviated any possibility that the 'gold standard' was influenced by ambient conditions, the thermistor tip being deep within white matter or ventricle. Whilst the IC consistently reflected the temporal changes in brain temperature during normothermia and during fever, suggesting a potential for the technique in clinical practice, the differences between brain and IC temperature were inconsistent; at times within $0.4^{\circ}$ or $0.5^{\circ} \mathrm{C}$ of brain but at other times too large (e.g. $1.0^{\circ} \mathrm{C}$ to $1.5^{\circ} \mathrm{C}$ ) to be clinically useful. Further studies are required to determine whether improvements in measurement technique would yield a better accuracy of the IR measurements to estimate brain temperature.

By undertaking the current pilot study we have discovered a novel potential for IR thermal imaging; a 'putative' role of the highest recorded IC temperature as a biomarker to predict 'sidedness' of the predominant TBI lesion. Here differences in temperature might be due to inflammation and higher arterial perfusion associated with the region of the lesion. Whilst this finding remains to be investigated further, it may be of potential clinical value in patients where CT imaging is not available. Studies are now ongoing in this area.

\section{Acknowledgements}

Our thanks go to our neurosurgical colleagues and the doctors and nurses of the intensive care unit, NUH for their continued support of our research. This work was supported via a grant support (CC) from the National University of Singapore.

\section{REFERENCES}

[1] Childs, C., Human brain temperature: regulation, measurement and relationship with cerebral trauma: Part 1. British Journal of Neurosurgery, 2008. 22(4): p. 486-496.

[2] Graf, W., Patterns of human liver temperature. Acta Physiologica Scand, 1959. 46(suppl (160)): p. 38-90.

[3] Romanovsky, A.A. and M. Szekely, Fever and hypothermia:two adaptive thermoregulatory responses to systemic inflammation. Medical Hypotheses, 1998. 50: p. 219-226.

[4] Busto, R., et al., Effect of mild hypothermia in ischaemic-induced release of neurotransmitters and free fatty acids in rat brain. Stroke, 1989. 20: p. 904-910.

[5] Childs, C., et al., Differences between brain and rectal temperature during routine critical care of patients with severe traumtic brain injury. Anaesthesia, 2005. 60(759-765).

[6] Childs, C., et al., Report of a consensus meeting on human brain temperatureafter severe traumatic brain injury: its measurement and managementduring pyrexia. Frontiers in Neurotrauma, 2010. 1(146): p. Published online 2010 November 23. doi: 10.3389/fneur.2010.00146.

[7] Kirk, D., et al., Infra-red thermometry: the reliability of tympanic and temporal artery readings fro predicting brain temperature after severe traumatic brain injury. Critical Care, 2009. in press. 
[8] Teunissen, L.P.J. and H.A.M. Daanen, Infrared thermal imaging of the inner canthus of the eye as an estimator of body core temperature. J. Medical Engineering \& technology, 2011. 35(3-4): p. 134-138.
[9] Erdogmus, S. and F. Govsa, Arterial features of inner canthus region: confirming the dafety for the flap region. The journal of craniofacial surgery, 2006. 17(5): p. 864-868 